\title{
The foundations of learning - a fresh perspective on common coaching methodologies
}

\author{
David Wilson (Ireland) \\ ITF Coaching and Sport Science Review 2015; 65 (23): 15-17
}

\begin{abstract}
Preparing players for elite competition requires a commitment to ongoing training and competition, both of which are underpinned by the knowledge a player has of the techniques/tactics necessary for success. The manner in which we teach these skills is crucial in determining the likely outcome when a player is tested under competition conditions. "Simplicity is the ultimate sophistication" Leonardo Da Vinci.
\end{abstract}

Key words: expertise, task, training, cognition, repetition Received: 25 November 2014 Accepted: 30 Januart 2015 Corresponding author: David Wilson Email: dwireland@me.com

\section{INTRODUCTION}

As the volume of data gathered in coaching research grows, so too does the pressure on coaches to implement new methodologies, new strategies and new ways of working with their players. Often however, this focus on finding bigger, better and more innovative ways of coaching can cause us to overlook the basic principles of player development and to therefore build our strategies on unstable foundations.

Critical to the success of long-term development is an understanding that skills learned during the junior years must be robust and deeply ingrained if they are to survive the extreme challenges of the professional stage. Ultimately, our training at junior level aims to develop players who can perform expert skills against significant opponents in extreme conditions and under immense pressure (Wilson, 2014).

To achieve this level of performance, it is vital to question the manner in which we currently conduct our training and practice sessions. While self-reporting from coaches and the content of coaching training manuals would suggest that a wide variety of strategies are employed, attempts to assess this have actually shown the opposite - That coaches tend to employ a very narrow range of coaching styles and that direct instruction remains the most commonly employed method of training tennis skills and strategies (Hewitt \& Edwards, 2013).

Preparing players for elite performance however, calls for the use of a number of core coaching fundamentals. While coaches are of course encouraged to be innovative in their approaches and to personalise their coaching strategies towards the specific needs of their own players, the following fundamentals provide an efficient base of effective learning.

\section{FUNDAMENTALS}

Understanding the task

The cornerstone of long-term learning is an ingrained understanding of a particular technique or skill. Unfortunately it is very difficult for a coach to fully assess how well a player understands the context of what is being taught. Equally, working with young players presents a further difficulty in that very often they will say that they understand something when in fact this is not the case.

In order to overcome these challenges a range of strategies can be employed. Asking the player to explain the content to another player for example, will highlight their own level of understanding. Actively questioning the player about the skill/tactic being trained will also help (in active questioning "yes" or "no" answers are not allowed - the player is required to fully explain their responses), as will an emphasis on regular self-reporting from the player during training.

There is no doubt that it takes time to confirm full understanding and that this may not be possible with groups of club or recreational players. For those aiming for elite status however, it is vital.

Source of Information

Direct instruction is characterised by the coach passing information to the player, who then trains this under supervision until a level of automation is achieved. While this strategy will tend to work in the short-term, the restrictions of 'explicit' learning are likely to appear when tested under pressure. At that stage the player will often deal with the stressful conditions presented to them by attempting to consciously and logically analyse each of the skills they need to implement. Unfortunately, focusing on the techniques of 'how to' perform a skill is unlikely to improve the stress of competition and will in fact very often detract from performance levels.

Instead, a focus on 'implicit' learning (Farrow, 2012) can protect technical skills from the stresses of elite competition. Implicit skills are learned without direct instruction from a coach and in contrast, are discovered by the player through a careful implementation of coaching strategies. Learning without direct instruction means that during high pressure competitive situations, players are unable to revert to a cognitive analysis of the stroke/strategy (because they don't have the initial instructional information to do that) and instead can focus on the task at hand of dealing with the opponent etc. 
Specific approaches for developing implicit learning include "errorless practice" (where we introduce a skill at a very easy level and build up), "guided discovery" (where a series of questions is used to enable the player to uncover the solution themselves), and "modeling" (where the player observes and replicates, without any verbal explanation being provided).

Cognitive Involvement

Although many of the technical/tactical requirements of elite tennis performance involve several distinct action steps (think for example of the many steps required to execute an attacking mid- court forehand), there is no need for the player themselves to be familiar with each and every one of these. Taking a player through the minute detail of each action he/she is required to learn and subsequently referring to many of these small points in feedback and analysis, points many times to a situation of 'cognitive overload'. This process of thinking through techniques and tactics during competition is inefficient and often buckles under stress (Muller \& Abernethy, 2012).

Coaches wishing to provide a more resilient form of learning devise methods of 'chunking' information, so that a series of actions or decisions which the player needs to learn are summarised in one phrase. These 'chunks' can be specific to the individual player and should be based on their playing competence and level of knowledge ("Follow the ball", "Drive up through the serve", etc.).

Chunking frees the mind to focus on first-hand issues, makes learning resilient under stress and allows for fast and effective feedback from coaches.

Efficiency of Repetition

A critical foundation of efficient learning is the extent and manner through which the skills and strategies learned are embedded through repetition. Again, while self-reporting from coaches suggests that a variety of training methods are used, anecdotal evidence would suggest that coaches tend to possess personal preferences for specific drill/practice methods and that these are used by them far more often than other options.

As one might imagine however, the use of a genuinely wide variety of practice types mirrors the varied and unpredictable nature of competitive tennis to a far greater extent than continuing to favor a small number of options.

To this end, practices should reinforce and test skills in a varied and variable manner using approaches such as game based coaching, basket feeding, closed drills, restricted court points, simulated points, etc. The crucial factor is that for learning to be assessed and reinforced, exclusive use of one or two practice strategies removes the potential gains available from other types. Core learning principles would suggest instead that coaches should implement a variety of practice approaches to stretch, test and challenge players in a difficult, unpredictable and dynamic fashion.

Learner State

Finally, basic learning fundamentals consistently highlight the need for those attempting to learn motor skills to be in the right frame of mind to do so (Fontana, 1993). Disinterested, demotivated or distracted players are of course far less likely to learn than those who are engaged, enthused and focused. To this end, coaches must always be conscious of the bigger picture when dealing with players.

Sport skill acquisition requires talent and a specific environment, and both need to coincide over a prolonged and purposeful period (Epstein, 2013). The timing of learning is therefore hugely important and coaches would be advised to implement technical/tactical training when distractions are at a minimum and when the player is open to the challenge of learning new skills.

The danger of 'over programming' (where the coach plans a series of weeks or months with very little flexibility for change) must clearly be avoided and instead the learning of new techniques/tactics should as much as possible be led by the player's wish to develop the new skill.

\section{CONCLUSIONS}

At a time when a multitude of information is available to coaches from publications, conferences and online sources, the basic foundations of learning are always worth re-emphasising. Although we may often think that by adding more and more teaching we are helping to improve our players (and indeed we may see evidence of this in practice), the fact remains that coaching has only been truly effective when the player can comfortably execute techniques/ tactics in competitive situations.

The core principles of coaching aim to make skill acquisition as easy as possible for the player and to develop techniques and tactics that are robust and capable of withstanding the stress of elite competition.

Recommendations

- Spend time building and checking full understanding from players. - Help players find their own solutions. Guide, don't instruct.

- Look for creative solutions. Use chunking to sum up and reinforce complex skills.

- In devising practice: Variety, variety, variety.

- Create the conditions that make learning possible, but motivation and desire should come from the player.

Making learning simple is hard, but the reasons are obvious, the work is engaging and the benefits are significant.

\section{REFERENCES}

Epstein, D. (2013). The Sport Gene: Inside the Science of Extraordinary Athletic Performance. Penguin.

Farrow, D. (2012). Implicit Learning: An alternative Approach to Instruction. Australian Institute of Sport, Volume 27 Number 1.

Fontana, D. (1993). Psychology for Teachers. BPS Books.

Hewitt, M. et Edwards, K. (2013). Observed teaching styles of junior development and club professional tennis coaches in Australia, ITF Coaching and Sport Science Review, 59, 6-8.

Mosston, M. et Ashworth, S. (1990). The Spectrum of Teaching Styles. From Command to Discovery. White Plains, NY: Longman.

Muller, S. et Abernethy, B. (2012). Expert Anticipatory Skill in Striking Sports. Research Quarterly for Exercise and Sport.https://doi.org/10.1080/02701367.2012.10 599848

Wilson, David (2014). Assessing the Benefits and Challenges of Common Coaching Methodologies in the Context of 14 and Under Learning Styles and Playing Competencies. Tennis Europe Coaches Conference, Tallinn. 


\section{Tennis@icoach}

RECOMMENDED ITF TENNIS ACADEMY CONTENT (CLICK BELOW)

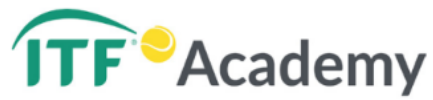

Copyright (c) David Wilson 2015

(c) (1)
This text is under a Creative Commons BY 4.0

license

You are free to Share - copy and redistribute the material in any medium or format - and Adapt the content - remix, transform, and build upon the material for any purpose, even commercially under the following terms:

Attribution: You must give appropriate credit, provide a tink to the license, and indicate if changes were made. You may do so in any reasonable manner, but not in any way that suggests the licensor endorses you or your use. 